\title{
BMJ Open Helminth infections, atopy, asthma and allergic diseases: protocol for a systematic review of observational studies worldwide
}

\author{
Margarete Arrais (D) , ,2 Tiago Maricoto, ${ }^{3,4}$ Philip Cooper, ${ }^{5,6}$ Jorge M R Gama, $^{7}$ \\ Bright I Nwaru, ${ }^{8}$ Miguel Brito, ${ }^{2,9}$ Luís Taborda-Barata (10) ${ }^{10,11}$
}

To cite: Arrais M, Maricoto T, Cooper P, et al. Helminth infections, atopy, asthma and allergic diseases: protocol for a systematic review of observational studies worldwide. BMJ Open 2020;10:e038085. doi:10.1136/ bmjopen-2020-038085

- Prepublication history and additional material for this paper are available online. To view these files, please visit the journal online (http://dx.doi org/10.1136/bmjopen-2020038085).

Received 26 February 2020 Revised 03 April 2020 Accepted 27 April 2020

Check for updates

(C) Author(s) (or their employer(s)) 2020. Re-use permitted under CC BY-NC. No commercial re-use. See rights and permissions. Published by BMJ.

For numbered affiliations see end of article.

Correspondence to

Professor Luís Taborda-Barata tabordabarata@fcsaude.ubi.pt

\section{ABSTRACT}

Introduction Childhood infections, particularly those caused by helminths are considered to be important environmental exposures influencing the development of allergic diseases. However, epidemiological studies focusing on the relationship between helminth infections and risk of allergic diseases, performed worldwide, show inconsistent findings. Previous systematic reviews of observational studies published 10 or more years ago showed conflicting findings for effects of helminths on allergic diseases. Over the past 10 years there has been growing literature addressing this research area and these need to be considered in order to appreciate the most contemporary evidence. The objective of the current systematic review will be to provide an up-to-date synthesis of findings of observational studies investigating the influence of helminth infections on atopy, and allergic diseases.

Methods and analysis This systematic review protocol was registered at PROSPERO. We will search Cochrane Library, MEDLINE, EMBASE, CINAHL, AMED, ISI Web of Science, WHO Global Health Library, Scielo, IndMed, PakMediNet, KoreaMed, Ichushi for published studies from 1970 to January 2020. Bibliographies of all eligible studies will be reviewed to identify additional studies. Unpublished and ongoing research will also be searched in key databases. There will be no language or geographical restrictions regarding publications. Critical Appraisal Skills Programme quality assessment tool will be used to appraise methodological quality of included studies. A descriptive summary with data tables will be constructed, and if adequate, meta-analysis using random-effects will be performed. The Preferred Reporting Items for Systematic Reviews and Meta-Analyses checklist will be followed for reporting of the systematic review.

Ethics and dissemination Since this systematic review will be only based on published and retrievable literature, no ethics approval will be sought. The multidisciplinary team performing this systematic review will participate in relevant dissemination activities. Findings will be presented at scientific meetings and publish the systematic review in international, peer-reviewed, openaccess journals.

PROSPERO registration number CRD42020167249.
Strengths and limitations of this study

- This is the first systematic review to address the relationship between a wide variety of helminth infections and atopy and the most frequent allergic diseases in children (ie, asthma, rhinitis and eczema).

- A thorough search strategy using leading databases in medicine and public health will ensure that the relevant papers on the topic will be identified.

- This protocol followed the Preferred Reporting Items for Systematic Review and Meta-Analysis Protocols guidelines.

- With no restriction by language or geography, this systematic review will provide a comprehensive and global picture on the topic and enables investigations into any regional differences.

- Different epidemiological definitions of allergic diseases, as well as the consideration of a wide variety of helminth parasite species for which numbers of eligible studies may be limited, may affect the validity of any subgroup meta-analyses by parasite species and allergic outcomes.

\section{INTRODUCTION}

Intestinal parasites include a large group of micro-organisms of which protozoans and helminths are of most relevance to human health. ${ }^{1}$ Most prevalent are the nematode helminth group, including Ascaris lumbricoides, Trichuris trichiura and hookworms (Necator americanus and Ancylostoma duodenale). ${ }^{2}$ Prevalence varies by geographic region and is greatest in endemic areas of sub-Saharan Africa, Latin America, China and Eastern Asia, where such infections are linked to poverty and poor sanitation. ${ }^{13-5}$

Allergic diseases such as asthma and rhinitis, and eczema affect millions worldwide. $^{6-8}$ Among environmental exposures considered to influence the development of allergic diseases are childhood infections including helminths. Helminths are capable 
of producing immunological mediators that modulate host immune responses, particularly allergic inflammation. ${ }^{9}{ }^{10}$ However, results of epidemiological studies addressing the relationship between helminth infections and risk of allergic diseases, done in almost all geographic regions of the world, show inconsistent findings. ${ }^{1-18}$

There are only two previous systematic reviews of observational studies investigating the relationship between helminth infections and allergy: (1) a systematic review included 30 studies published up to 2006 in a metaanalysis of the relationship between helminths and asthma symptoms and showed overall no association but parasite-specific effects were observed-hookworm was associated with a reduced while $A$. lumbricoides was associated with an increased risk of symptoms ${ }^{19} ;(2)$ a systematic review of 21 observational studies published up to 2009, studied the relationship between helminths and allergen skin prick test (SPT) reactivity and showed an inverse association between helminths and $\mathrm{SPT}^{20}$ Three other more recent systematic reviews have studied the relationship between Toxocara spp seroprevalence and allergy: (1) a meta-analysis from 2013 of 10 studies addressing the association between Toxocara spp and asthma showed an increased risk ${ }^{21}$; (2) a systematic review from 2017 concluded that children infected with Toxocara spp were more likely to have asthma ${ }^{22}$ and (3) a study from 2018 showed that Toxocara spp was associated with an increased risk of urticaria but not with atopy or eczema. ${ }^{23}$

Existing systematic reviews and meta-analyses investigating the role of various helminth intestinal parasites in atopy or allergic diseases are 10 or more years old and considered few allergic outcomes (eg, asthma or atopy). As detailed above, more recent systematic reviews have focused only on Toxocara spp infections. Given these important gaps and diverging results in the literature, the aims of the current systematic review are to identify, critically appraise and synthesise the evidence from observational epidemiological studies investigating the influence of various species and parasite burdens of helminth infections on the: (1) risk of developing asthma, rhinitis, eczema and/or atopy and (2) the expression of clinical outcomes and disease severity in patients with already established allergic diseases. Because of the recent systematic reviews addressing toxocariasis, Toxocara spp will not be considered here.

\section{METHODS AND ANALYSIS}

This study has been registered with the International prospective register of systematic reviews (PROSPERO). The review will be reported in accordance with the Preferred Reporting Items for Systematic Reviews and Meta-Analyses (PRISMA) guidelines for systematic reviews and MOOSE (Meta-analysis of Observational Studies in Epidemiology) guidelines for meta-analysis of observational epidemiological studies. ${ }^{24}{ }^{25}$ Any modifications in the protocol during the systematic review will be reported.

\section{Search strategy}

We have developed a comprehensive search strategy for retrieving published and unpublished studies on the topic (online supplementary appendix 1). We will search the Cochrane Library (Cochrane Database of Systematic Reviews, Cochrane Central Register of Controlled Trials (CENTRAL), Cochrane Methodology Register), MEDLINE, EMBASE, CINAHL, AMED, ISI Web of Science (Science and Social Science Index), WHO Global Health Library (which encompasses African Index Medicus, Index Medicus for the Eastern Mediterranean Region, Index Medicus for the South-East Asia, Latin America and the Caribbean Literature on Health Sciences and Western Pacific Region Index Medicus), Scielo, IndMed, PakMediNet, KoreaMed and Ichushi (updated by Japan Medical Abstracts Society). Search dates will be from 1970 (or from the inception of a database if this occurs later than 1970) to present. The bibliographies of all eligible studies will be reviewed to identify additional possible studies. We will identify unpublished and ongoing work and research in progress by searching key Internet-based relevant databases (www.clinicaltrials.gov; www.clinical trialsregister.eu; www.controlledtrials.com; www.anzctr. org.au). In addition, we will contact authors who have published in this field to ask for potentially additional papers. No language restrictions will be imposed; translations will be undertaken where necessary.

\section{Inclusion criteria for study designs}

We will include all observational and analytical epidemiological studies, including cohort, case-control and crosssectional studies.

We will select all the studies that include participants of any age, in which the relationship between helminth infection and respiratory allergic diseases, atopic eczema and/or atopy has been studied and include studies investigating any type of helminth infection, including Enterobius vermicularis, A. lumbricoides, T. trichiura, hookworm (A. duodenale and N. americanus), Strongyloides stercoralis, Hymenolepsis spp (H. nana and H. diminuta) and Schistosoma $\operatorname{spp}(S$. mansoni and S. haematobium). Toxocara $\mathrm{spp}$ infections will not be considered in this review given the recent systematic review that considered it. Where applicable, the comparator to helminth infection will be no infection. Some studies would have compared different types of helminth infections and load of infection; we will maintain these comparisons in the systematic review.

We will exclude discussion papers, non-research letters and editorials, randomised controlled trials, clinical case studies and case-series, and animal studies.

\section{Study selection}

Papers retrieved from the databases will be exported to a reference management programme where further screening will be undertaken. Removal of duplicate publications will be performed, thereafter, the titles and abstracts of retrieved papers will be checked by two investigators. The full text of all potentially eligible studies 
will be retrieved and independently assessed against the inclusion criteria (see above) by two reviewers. The reviewers will decide which of the studies fit the inclusion criteria. Any disagreements will be resolved by discussion, with a third reviewer arbitrating in the circumstance of unresolved discrepancies.

To ensure transparency, the process of selection will be summarised using a PRISMA flow diagram.

\section{Data extraction and management}

Data from selected articles will be transferred from their original presentation to a proper form made in Microsoft Excel software, with each study receiving a reference code. Each study will have its own extraction form. If necessary, we will collect indirect data from figures and charts, adapting their interpretation from two different authors by consensus and authors of original articles will also be contacted for further information and data.

For all included studies, we will be collect the following information: study design, number of participants and their characteristics (namely, wheezing due to early-life respiratory viral infections, early childhood respiratory infections, personal and family history of allergies, household smoking), country of study, year of publication, profiles of helminth infection (presence, load, duration of infection, types of parasites, monoinfection or coinfection, recent or ancient treatment, frequency of infection), geographical differences; estimates (HR, risk ratio, OR, 95\% CIs, mean and SD) of the association between helminth infection and the study outcomes; confounding factors will also be analysed, if reported, namely malaria, tuberculosis or HIV coinfection, technical aspects of determination/operational definition of helminth infection.

Data extraction will be completed independently by two reviewers and discrepancies will be decided by a third reviewer.

\section{Outcomes}

\section{Primary outcome}

Estimates of association between helminth infection and incidence of asthma (either doctor diagnosed or wheeze in the past 12 months-eg, ISAAC (International Study of Asthma and Allergies in Childhood) study definition or other comparable definitions), allergic rhinitis (doctor diagnosed or as defined in the ISAAC study or other comparable definitions), eczema (flexural dermatitis diagnosed by doctor or as defined in the ISAAC study or other comparable definitions) and atopy (assessed using allergen-specific IgE or SPTs).

\section{Secondary outcome}

Estimates of association between helminth infection and clinical outcomes of respiratory allergic diseases, including exacerbations, hospitalisations, severity according to clin$\mathrm{ical} /$ symptoms evaluation (using any type of validated scale or questionnaire) and health-related quality of life (using any type of validated scale or questionnaire).

\section{Quality assessment}

Risk of bias assessment will be independently undertaken by two different reviewers, using the Critical Appraisal Skills Programme ${ }^{26}$ quality assessment tool for the types of included studies. We will appraise different components of each study, including appropriateness of study design, potential for selection bias, measurement of exposures and outcomes and generalisability of the study findings. For each study, the grading of each individual components and the global study rating will be assigned categories of risk of bias: low, moderate and high. The global grading will involve taking an average of all individual components. Any disagreements not resolved by discussion will be arbitrated by a third reviewer.

\section{Quantitative assessment}

If necessary, and according to the available reported data on risk associations, a quantitative analysis will be performed to obtain effect estimations, heterogeneity and consistency tests. Forest plots will be used to graphically present the results of the meta-analysis. Funnel plots will be used to graphically assess small study effect, which is one type of publication bias. Heterogeneity between effect sizes of included studies will be assessed by visual inspection of forest plots and by using the $\chi^{2}$ test for heterogeneity (with a $\mathrm{p}$ value of $<0.1$ ) and inconsistency between studies will be described using the percentage of the variability in effect estimates that is due to heterogeneity rather than chance $\left(\mathrm{I}^{2}\right)$. It is generally accepted that $\mathrm{I}^{2}$ values up to $25 \%, 50 \%$ and $75 \%$ represent low, medium and high levels of heterogeneity or inconsistency, respectively, ${ }^{27}$ although it is now clear that this statistics is not an absolute measure of such heterogeneity and rather indicates the proportion of observed variance that reflects variance in true effect sizes rather than sampling error. ${ }^{29}$ We plan to use Mantel-Haenzsel risk ratios with a random-effects model and 95\% CI for dichotomous data. Continuous outcomes (such as symptoms or quality of life assessments) will be analysed as standardised mean difference values using a random-effects model and $95 \% \mathrm{CI}$, because the included studies may report different measurement instruments. We also plan to performed sensitivity analysis of the included studies and their impact on meta-analysis. If results from longitudinal studies allow, the primary outcomes (such as risk ratios or HRs) will also be analysed with an adaptation form trial sequential analysis using O'Brien Fleming monitoring boundaries approach, in order to avoid false positive or negative results of the pooled meta-analysis, thereby avoiding false or negative results. ${ }^{30}$ Subgroup analysis may be performed according to different reported species of helminths or diseases, rural versus urban settings, age groups, geographical regions and by study design.

\section{Data synthesis}

We will produce a descriptive summary table of all included studies in order to summarise the literature. For studies without required data (eg, relative risk estimates 
of effect of helminth infection and the outcomes), we will undertake a narrative synthesis of the data in which we use texts to describe the overall findings from the studies, highlight their strengths and weaknesses and make textual comparisons between the studies in this category in the light of the study question. For studies we judge to be reasonably clinically and methodologically homogeneous (ie, have used similar methods with regards to subject selection and inclusion, helminth definition and assessment, outcome definition and assessment and statistical analyses), we will perform meta-analyses using random-effects models to estimate the combined effect of helminth infection on each of the study outcome. The meta-analysis for the association between helminth infection and each outcome will be undertaken separately. We will quantify the heterogeneity between studies using the $\mathrm{I}^{2}$ statistic, which is a measure (range $0 \%-100 \%$ ) used to quantify the proportion of variance in the pooled estimates attributable to differences in estimates between studies included in the meta-analysis. ${ }^{27-30}$ The betweenstudy variance will be estimated using the Tau-squared $\left(\tau^{2}\right)$ statistic derived from the DerSimonian-Laird approach. ${ }^{31}$ Where data are available, we will perform subgroup analyses according to rural versus urban settings, age groups, geographical regions of the world and by other potential characteristics, such as study design (cohort, case-control, cross-sectional studies). We will perform sensitivity analyses based on the sample size of included studies as well as on the basis of the risk of bias results in the studies in order to assess the robustness of our findings to different assumptions. In the case of sensitivity analysis on the basis of study quality, we will estimate the combined effect estimates from all studies regardless of their quality grading (low, moderate, high). Then we will exclude all low-quality studies, leaving the moderate and high-quality studies; we will then compare the results to the results when all studies were combined regardless of their quality. We will repeat the process by excluding the moderate quality studies, leaving only the high-quality studies and then we will compare the results to those from the previous results. We will assess evidence of publication bias using funnel plots and statistically using Begg and Egger tests. ${ }^{32} 33$ The meta-analyses will be performed using Stata statistical software (release V.13; StataCorp LP., College Station, Texas, USA). The PRISMA checklist will be followed for reporting of the systematic review.

\section{Ethics and data management plan}

No ethical approval required because the data to be collected and analysed will be based only on the published literature and therefore cannot be linked to specific individuals. Retrieved data will be kept in a database that will have protected access and will only be used by the involved authors. However, anonymised data will be placed in an open repository.

\section{Patient and public involvement}

Since this will be a systematic review, there will be no direct patient or public involvement.

\section{ETHICS AND DISSEMINATION}

This systematic review will allow us, for the first time, to synthesise the findings of observational studies addressing the associations between a wide variety of relevant helminth parasites and common allergic outcomes. The review will be based on publications published between 1970 and January 2020, and will allow us to analyse methodological aspects of selected studies namely study design, regarding the questions, methods used and risk of selection bias.

More specifically, our review will fill in an important gap since previous systematic reviews are either dated or focused on a single helminth (eg, Toxocara). Thus, our study will provide relevant up-to-date information on current knowledge of helminth-allergy associations in children and adults. This will be done by accessing information worldwide without geographical or language restrictions in which; (1) various parameters related to helminth infection will be analysed-types of helminths, infection load, frequency of infections, among other; (2) the relationship will be analysed between helminth parameters and not just a single allergic disease but on a broader context of atopy, asthma, allergic rhinitis and eczema and (3) the relationship will not only be analysed in terms of risk of disease development but also regarding disease severity.

We believe our results should allow us to draw meaningful conclusions about the relevance and type of effects involved in the relationship between helminth infections and atopy, asthma and allergic diseases in children and adults, and may have clinical and societal implications.

Our dissemination strategy will involve presentations at scientific meetings, as well as publication of article(s) in international, peer-reviewed, open-access journals. However, given the increasing relative percentage of children with atopy, asthma and allergic diseases worldwide, particularly in certain geographical areas, the relevant burden of helminth infections in certain parts of the world, we also plan to organise meetings with general practitioners and other healthcare providers, as well as with local communities (namely in Africa) to analyse and discuss our findings and their potential implications.

\section{Author affiliations}

${ }^{1}$ Department of Pulmonology, Military Hospital Luanda, Luanda, Angola

${ }^{2} \mathrm{CISA}$ - Health Sciences Research Center of Angola, Caxito, Bengo, Angola

${ }^{3} \mathrm{ACeS}$ Baixo Vouga, Aveiro-Aradas Family Health Unit, Aveiro, Portugal

${ }^{4}$ Faculty of Health Sciences, University of Beira Interior, Covilhã, Portugal

${ }^{5}$ Institute of Infection and Immunity, St George's University of London, London, UK

${ }^{6}$ School of Medicine, International Univeristy of Ecuador, Quito, Ecuador

${ }^{7}$ Centre of Mathematics and Applications, Faculty of Sciences, University of Beira Interior, Covilhã, Portugal

${ }^{8}$ Krefting Research Centre, Institute of Medicine, Sahlgrenska Academy, University of Gothenburg, Gothenburg, Sweden 
${ }^{9}$ Health and Technology Research Center (H\&TRC), Lisbon Higher School of Health Technology, Polytechnic Institute of Lisbon, Lisbon, Portugal

${ }^{10}$ Department of Allergy \& Clinical Immunology, Cova da Beira University Hospital

Centre, Covilhã, Portugal

${ }^{11}$ CICS - Health Sciences Research Centre, University of Beira Interior, Covilhã, Portugal

Acknowledgements The authors would like to acknowledge Professor João Costa, of the Portuguese Collaborating Center of the IberoAmerican Cochrane Network, Faculty of Medicine, University of Lisbon, Portugal, for his willingness to assist with planned searches for this systematic review in Cochrane Library.

Contributors All authors contributed to the design and conceptualisation of this review. MA drafted the protocol with primary support from BIN (review guarantor) and LT-B. TM, PC, JMRG, BIN, MB and LT-B were involved in checking various steps of the search strategy, including keywords, as well as the final version of the protocol. JMRG was involved in the statistical strategy for data analysis. MA, TM, JMRG and LT-B were involved in establishing eligibility criteria and data extraction forms. All authors provided feedback on the manuscript, at all stages.

Funding The authors have not declared a specific grant for this research from any funding agency in the public, commercial or not-for-profit sectors.

Competing interests None declared.

Patient and public involvement Patients and/or the public were not involved in the design, or conduct, or reporting, or dissemination plans of this research.

Patient consent for publication Not required.

Provenance and peer review Not commissioned; externally peer reviewed.

Open access This is an open access article distributed in accordance with the Creative Commons Attribution Non Commercial (CC BY-NC 4.0) license, which permits others to distribute, remix, adapt, build upon this work non-commercially, and license their derivative works on different terms, provided the original work is properly cited, appropriate credit is given, any changes made indicated, and the use is non-commercial. See: http://creativecommons.org/licenses/by-nc/4.0/.

\section{ORCID iDs}

Margarete Arrais http://orcid.org/0000-0001-6611-4260

Luís Taborda-Barata http://orcid.org/0000-0001-6649-8890

\section{REFERENCES}

1 Harhay MO, Horton J, Olliaro PL. Epidemiology and control of human gastrointestinal parasites in children. Expert Rev Anti Infect Ther 2010;8:219-34.

2 World Health Organization. Soil-transmitted helminth infections. Available: https://www.who.int/news-room/fact-sheets/detail/soiltransmitted-helminth-infections [Accessed 30 Nov 2019].

3 Soares Magalhães RJ, Langa A, Pedro JM, et al. Role of malnutrition and parasite infections in the spatial variation in children's anaemia risk in northern Angola. Geospat Health 2013;7:341-54.

4 Abdi M, Nibret E, Munshea A. Prevalence of intestinal helminthic infections and malnutrition among schoolchildren of the Zegie Peninsula, northwestern Ethiopia. J Infect Public Health 2017;10:84-92.

5 de Alegría MLAR, Colmenares K, Espasa M, et al. Prevalence of Strongyloides stercoralis and Other Intestinal Parasite Infections in School Children in a Rural Area of Angola: A Cross-Sectional Study. Am J Trop Med Hyg 2017;97:1226-31.

6 Morales E, Strachan D, Asher I, et al. Combined impact of healthy lifestyle factors on risk of asthma, rhinoconjunctivitis and eczema in school children: Isaac phase III. Thorax 2019;74:531-8.

7 The Global Asthma Report. Global asthma network, 2018. Available: http://globalasthmareport.org/ [Accessed Oct 2019].

8 Chinratanapisit S, Suratannon N, Pacharn P, et al. Prevalence and severity of asthma, rhinoconjunctivitis and eczema in children from the Bangkok area: the global asthma network (GAN) phase I. Asian Pac J Allergy Immunol 2019;37:226-31.

9 Cooper PJ, Chico ME, Rodrigues LC, et al. Reduced risk of atopy among school-age children infected with geohelminth parasites in a rural area of the tropics. J Allergy Clin Immunol 2003;111:995-1000.
10 Cooper PJ, Chico ME, Guadalupe I, et al. Impact of early life exposures to geohelminth infections on the development of vaccine immunity, allergic sensitization, and allergic inflammatory diseases in children living in tropical Ecuador: the ECUAVIDA birth cohort study. BMC Infect Dis 2011;11:184.

11 Cooper PJ, Chico ME, Bland M, et al. Allergic symptoms, atopy, and geohelminth infections in a rural area of Ecuador. Am J Respir Crit Care Med 2003;168:313-7.

12 Dagoye D, Bekele Z, Woldemichael K, et al. Wheezing, allergy, and parasite infection in children in urban and rural Ethiopia. Am J Respir Crit Care Med 2003;167:1369-73.

13 Haileamlak A, Dagoye D, Williams $\mathrm{H}$, et al. Early life risk factors for atopic dermatitis in Ethiopian children. J Allergy Clin Immunol 2005;115:370-6.

14 van den Biggelaar AHJ, Rodrigues LC, van Ree R, et al. Long-Term treatment of intestinal helminths increases mite skin-test reactivity in Gabonese schoolchildren. J Infect Dis 2004;189:892-900.

15 Palmer LJ, Celedón JC, Weiss ST, et al. Ascaris lumbricoides infection is associated with increased risk of childhood asthma and atopy in rural China. Am J Respir Crit Care Med 2002;165:1489-93.

16 Lynch NR, Palenque M, Hagel I, et al. Clinical improvement of asthma after anthelminthic treatment in a tropical situation. Am J Respir Crit Care Med 1997;156:50-4.

17 Davey $\mathrm{G}$, Venn A, Belete $\mathrm{H}$, et al. Wheeze, allergic sensitization and geohelminth infection in Butajira, Ethiopia. Clin Exp Allergy 2005;35:301-7.

18 Cooper PJ, Chico ME, Vaca MG, et al. Effect of albendazole treatments on the prevalence of atopy in children living in communities endemic for geohelminth parasites: a clusterrandomised trial. Lancet 2006;367:1598-603.

19 Leonardi-Bee J, Pritchard D, Britton J. Asthma and current intestinal parasite infection: systematic review and meta-analysis. Am J Respir Crit Care Med 2006;174:514-23.

20 Feary J, Britton J, Leonardi-Bee J. Atopy and current intestinal parasite infection: a systematic review and meta-analysis. Allergy 2011;66:569-78.

21 Li L, Gao W, Yang X, et al. Asthma and toxocariasis. Ann Allergy Asthma Immunol 2014;113:187-92.

22 Aghaei S, Riahi SM, Rostami A, et al. Toxocara spp. infection and risk of childhood asthma: a systematic review and meta-analysis. Acta Trop 2018;182:298-304.

23 Mohammadzadeh I, Riahi SM, Saber V, et al. The relationship between Toxocara species seropositivity and allergic skin disorders: a systematic review and meta-analysis. Trans $R$ Soc Trop Med Hyg 2018;112:529-37.

24 Moher D, Shamseer L, Clarke M, et al. Preferred reporting items for systematic review and meta-analysis protocols (PRISMA-P) 2015 statement. Syst Rev 2015;4:1.

25 Stroup DF, Berlin JA, Morton SC, et al. Meta-Analysis of observational studies in epidemiology: a proposal for reporting. meta-analysis of observational studies in epidemiology (moose) group. JAMA 2000;283:2008-12.

26 Critical Appraisal Skills Programme (2018). Casp (checklist systematic review), 1994. Available: https://casp-uk.net/wp-content/ uploads/2018/01/CASP-Systematic-Review-Checklist_2018.pdf [Accessed 31 Jan 2020].

27 Higgins JPT, Thompson SG. Quantifying heterogeneity in a metaanalysis. Stat Med 2002;21:1539-58.

28 Higgins JPT, Thompson SG, Deeks JJ, et al. Measuring inconsistency in meta-analyses. BMJ 2003;327:557-60.

29 Borenstein M, Higgins JPT, Hedges LV, et al. Basics of meta-analysis: $\mathrm{I}^{2}$ is not an absolute measure of heterogeneity. Res Synth Methods 2017;8:5-18.

30 Wetterslev J, Thorlund K, Brok J, et al. Trial sequential analysis may establish when firm evidence is reached in cumulative meta-analysis. $J$ Clin Epidemiol 2008;61:64-75.

31 Higgins JPT, Thompson SG, Spiegelhalter DJ. A re-evaluation of random-effects meta-analysis. J $R$ Stat Soc Ser A Stat Soc 2009;172:137-59.

32 Begg CB, Mazumdar M. Operating characteristics of a RANK correlation test for publication bias. Biometrics 1994;50:1088-101.

33 Egger M, Davey Smith G, Schneider M, et al. Bias in meta-analysis detected by a simple, graphical test. BMJ 1997;315:629-34. 